\title{
Eficiencia de un amortiguador de columna de líquido considerando una excitación sísmica de alto contenido de frecuencias e incertidumbre
}

\section{Efficiency of a tuned liquid column damper considering a seismic excitation of high frequency} content and uncertainty

Fecha de entrega: 19 de octubre 2020 Fecha de aceptación: 31 de marzo 2021

\section{Gilda Espinoza, Juan Saavedra, Kristel Gajardo, Álvaro Suazo y Carlos Cifuentes}

Departamento de Ingeniería Civil y Ambiental, Universidad del Bío-Bío, Avenida Collao 1202, Casilla 5-C, Concepción, Chile, gespinoz@ubiobio.cl, juan.saavedra.ma@gmail.com,kgajardo@egresados.ubiobio.cl, asuazo@ubiobio.cl,

cacifue@egresados.ubiobio.cl

En esta investigación se estudia el comportamiento de una estructura de un grado de libertad sometida a una excitación sísmica estocástica de alto contenido de frecuencias, la cual es controlada por un amortiguador de columna de líquido sintonizado (ACLS) óptimo, cuando se introduce incertidumbre en algunos parámetros del sistema. Se analiza la influencia en el comportamiento de los parámetros óptimos del ACLS, en la eficiencia medida como reducción del desplazamiento del sistema principal y en la raíz cuadrática media (RMS) de desplazamiento de la estructura. Se realiza una linealización estadística equivalente para incluir la naturaleza no lineal del ACLS. Los parámetros óptimos de ACLS son la razón de frecuencias y el coeficiente de pérdida de carga. La optimización del ACLS se realiza minimizando la reducción de la desviación estándar del desplazamiento de la estructura principal. La incertidumbre es ingresada en la razón de masa, la razón de longitud y el coeficiente de pérdida de carga. Se analiza el comportamiento de la eficiencia, medida como una reducción porcentual del desplazamiento al incluir un ACLS óptimo y el RMS del desplazamiento, ante la inclusión de incertidumbre en los parámetros antes mencionados. Se encuentra que la introducción de incertidumbre en el periodo de la estructura produce la mayor sensibilidad en los parámetros óptimos del ACLS, la eficiencia y el RMS del desplazamiento de la estructura. Esto se produce principalmente en estructuras rígidas y con una razón de masa pequeña. Por otra parte, una incertidumbre en la razón de masa, y en la razón de longitud del ACLS, no produce variaciones en la eficiencia ni en el RMS de desplazamiento de la estructura.

Palabras clave: ACS, ACLS, incertidumbre en los parámetros, análisis estocástico, alto contenido de frecuencia
In this research the behaviour of a one degree of freedom structure controlled by an optimal tuned liquid column damper (TLCD) and subjected to stochastic seismic excitations of high frequency content is studied. Uncertainty is introduced in some system parameters. The influence on the behaviour of the optimal TLCD parameters, on the efficiency measured as reduction of main system displacement and on the root mean square (RMS) displacement of the structure is analysed. An equivalent statistical linearization is performed to include the non-linear nature of the TLCD. The optimal TLCD parameters are the frequency ratio and the pressure drop coefficient. TLCD optimization is performed by minimizing the standard deviation reduction of mainframe displacement. The uncertainty is entered into the mass ratio, length ratio, and head loss coefficient. The behaviour of the efficiency is analysed, measured as a percentage reduction of the displacement when including an optimal TLCD and the RMS of the displacement, before the inclusion of uncertainty in the aforementioned parameters. It is found that the introduction of uncertainty in the period of the structure produces the highest sensitivity in the optimal parameters of the TLCD, the efficiency and the RMS of the displacement of the structure. This occurs mainly in rigid structures and with a small mass ratio. On the other hand, an uncertainty in the mass ratio, and length ratio of the TLCD does not produce variations in the efficiency or in the RMS of displacement of the structure.

Keywords: TLCD, parameter uncertainty, stochastic analysis, high frequency content 


\section{Introducción}

El diseño de estructuras se debe hacer bajo la ley de diseño sismorresistente, C (capacidad) $>$ D (demanda). Tradicionalmente esta ecuación se puede satisfacer a través del aumento de la capacidad de la estructura. Por medio de otorgar suficiente resistencia para resistir solicitaciones, además de proporcionar seguridad y serviciabilidad a las personas que se encuentren en ellas. En Chile las excitaciones son de alto contenido de frecuencias. Esto queda plasmado en el espectro de diseño de la norma chilena NCh433 (2009). En los últimos treinta años, se han generado nuevas metodologías y sistemas dinámicos con el objetivo de reducir la ecuación capacidad versus demanda a través de la reducción de la demanda por medio de la modificación de las características dinámicas de las estructuras al incluir un dispositivo de protección sísmica. Dentro de los estudios que se han realizado, se encuentran los sistemas de efecto masa, en los que se distinguen dos tipos, el amortiguador de masa sintonizado AMS y el amortiguador de columna de líquido sintonizado ACLS.

El ACLS es un tubo en forma de U que contiene líquido en su interior, generalmente agua y en su parte inferior horizontal posee un orificio que genera una pérdida de carga (Chakraborty et al., 2012). Uno de los primeros estudios corresponde a Balendra et al. (1995), quienes estudian la eficiencia de los ACLS en el control de las vibraciones inducidas por viento para una gama torres con distintas frecuencias. Concluyen que el desempeño del ACLS no depende sólo de la razón de sintonía, sino también de la razón de apertura del orificio.

Shum y Xu (2002) realizan un ensayo experimental para analizar el rendimiento de múltiples amortiguadores de columna de líquido óptimos (MACLS) en el control de torsión en una estructura. Construyen un modelo de acero, el que es solicitado por vibraciones armónicas forzadas en la base de la estructura, por medio de una plataforma de prueba. Los resultados arrojan que los MACLS poseen un mayor rendimiento que un ACLS con la misma cantidad de agua. Desarrollan también ecuaciones para un sistema MACLS-estructura bajo excitaciones basales armónicas o del tipo ruido blanco y comparan los resultados con la investigación experimental.

Shum (2009) desarrolla un esquema de fórmula cerrada para obtener los parámetros óptimos de diseño de un ACLS, para el caso de una estructura principal no amortiguada. Optimizan la respuesta a través de dos puntos invariantes, validando su propuesta a través de la comparación de resultados con el método iterativo convencional.

Lee et al. (2010) estudian experimentalmente la variación de las características dinámicas del ACLS, respecto a excitaciones de distinta amplitud. Concluyen que la frecuencia natural, factor de amortiguamiento, relación entre la masa de líquido y la masa de líquido horizontal afectan el comportamiento dinámico del ACLS.

Investigaciones más recientes realizadas por Zhu et al. (2015) plantean la utilización de la técnica de simulación híbrida en tiempo real, con un prototipo de un ACLS, que es modelado como una subestructura física y sometido a excitaciones basalesa través de unamesa vibratoria,mientras que la estructura principal se simula numéricamente en un computador. Con esta técnica estudian la eficiencia del ACLS, en la reducción de las respuestas modales. Di Matteo et al. (2015) proponen una ecuación diferencial fraccional del movimiento, que describe con precisión el movimiento del líquido en el contenedor, comparan su propuesta con pruebas experimentales.

Otras investigaciones modifican el diseño del ACLS con el objetivo de obtener una mayor eficiencia, dentro de estas se destaca la realizada por Rozas et al. (2016), quienes proponen un ACLS bidireccional, compuesto por dos ACLS ubicados en dirección ortogonal. Concluyen que este tipo de configuración disminuye la respuesta ante eventos sísmicos en dos direcciones perpendiculares, requiriendo una menor masa de agua con respecto a dos ACLS equivalentes.

Espinoza et al. (2018) analizan el comportamiento de una estructura no lineal a la cual se le añade un ACLS óptimo ante excitaciones sísmicas estocásticas. La optimización del ACLS se realiza minimizando el desplazamiento del sistema estructural, la reducción de la energía histerética y un ponderado de ambos. Concluyen que el ACLS se sintoniza con la segunda frecuencia lineal equivalente, cuando la excitación es de ancho de banda amplio, y se sintoniza con la frecuencia predominante del input cuando la excitación es de ancho de banda angosto.

Pabarja et al. (2019) investigan la eficiencia de un ACLS 
óptimo en el control de estructuras con irregularidad vertical. Estudian el comportamiento de una estructura de tres pisos, cuando es sometida a pruebas de vibración libre y forzada. Concluyen que, existen diferencias con el caso de estructuras regulares, si se diseña un ACLS en la forma tradicional.

Debbarma et al. (2010) investigan el comportamiento de una estructura cuando se introduce incertidumbre en los parámetros de una estructura controlada por un ACLS óptimo. La incertidumbre se realiza mediante un desarrollo en serie de Taylor, respecto a los parámetros con incertidumbre, de la matriz de covarianza y de las matrices de espacio de estado, en la ecuación de Lyapunov.

Espinoza et al. (2020a,b) analizan la eficiencia del ACS, el que consiste en un amortiguado de masa sintonizado (AMS) unido a un amortiguador de líquido sintonizado (ACLS). Se ingresa incertidumbre en los parámetros sometidos a excitaciones sísmicas del alto contenido de frecuencias (Espinoza et al., 2020a) y bajo contenido de frecuencias (Espinoza et al., 2020b). Los resultados más importantes muestran que en el caso de sismos de alto contenido de frecuencias, el parámetro más sensible es el coeficiente de pérdida de carga, ante una incertidumbre en la razón de longitud. Y en el caso de bajo contenido de frecuencias, la eficiencia del ACS es máxima cuando el periodo de la estructura coincide con el periodo predominante de la excitación sísmica.

Se concluye que la eficiencia del ACS y RMS de desplazamiento de la estructura principal, es insensible a una incertidumbre menor a un $10 \%$, tanto de la razón de masa y de la razón de longitud.

Debbarma et al. (2010) no analizan la eficiencia de un ACLS sobre una estructura cuando es sometida a una excitación aleatoria de alto contenido de frecuencia típica de Chile. Por lo tanto, el objetivo de esta investigación, es analizar el comportamiento de un ACLS óptimo en una estructura controlada, cuando es sometida a excitaciones sísmicas de alto contenido de frecuencias, compatibles con sismo chileno derivado del espectro chileno NCh 2745 (2013), considerando incertidumbre en los parámetros del sistema. El caso de excitación sísmica con bajo contenido de frecuencias con incertidumbre es analizado en Espinoza et al. (2021).

\section{Modelo estructural y ecuaciones de movimiento}

\section{Modelo estructural}

La Figura 1 muestra el modelo de estudio M1. El modelo M1 consiste en una estructura lineal de un grado de libertad $x_{s}$, a la que se le ha adicionado un amortiguador de columna de líquido sintonizado ACLS. Este es un tubo en forma de U con agua en su interior y posee un orificio en la parte inferior horizontal. Las variables que se consideran en el modelo son la rigidez elástica $k_{s}$, amortiguamiento $c_{s}$ y la masa de la estructura principal $m_{s}$. Los parámetros del ACLS son la longitud de la parte horizontal del tubo $b_{h}$, altura de la columna de líquido $h$; $x$ es el desplazamiento de la estructura principal en el eje horizontal, $y$ es el desplazamiento del líquido dentro de la columna en el eje vertical. La aceleración del suelo es expresada como $\ddot{u}_{g}$.

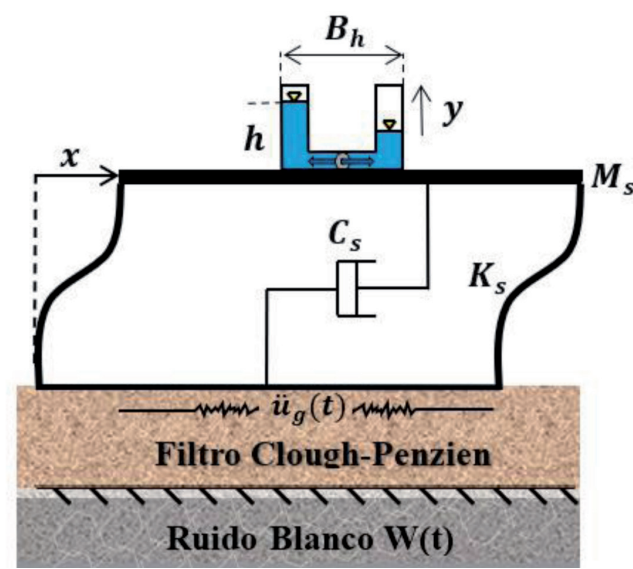

Figura 1: Modelo del estudio

\section{Ecuaciones de movimiento de $M_{1}$}

La ecuación (1) corresponde a la ecuación del movimiento de un sistema con un ACLS sometida a una aceleración vertical (Sakai et al., 1989):

$\rho A L_{e} \ddot{y}+\frac{1}{2} \rho \xi A|\dot{y}| \dot{y}+2 \rho g A y=-\rho A B_{h}\left(\ddot{x}+\ddot{u}_{g}\right)$

donde $L_{e}=2 h+B_{h}$ es la longitud efectiva de la columna líquida, $\rho$ es la densidad del líquido contenida por el ACLS, $A$ es el área de la sección transversal y $g$ es la aceleración de gravedad. El coeficiente de pérdida de carga corresponde a $\xi$, controlado por el diámetro del orificio de la porción horizontal del ACLS. La ecuación (1) es linealizada estadísticamente, quedando como la ecuación (2). 


$$
\rho A L_{e} \ddot{y}+2 \rho A c_{p} \dot{y}+2 \rho g A y=-\rho A B_{h}\left(\ddot{x}+\ddot{u}_{g}\right)
$$

$$
\begin{aligned}
& {\left[\begin{array}{cc}
1 & p \\
\mu p & (1+\mu)
\end{array}\right]\left\{\begin{array}{l}
\ddot{y} \\
\ddot{x}
\end{array}\right\}+\left[\begin{array}{cc}
\frac{2 c_{p}}{L_{e}} & 0 \\
0 & 2 \xi_{s} \omega_{s}
\end{array}\right]\left\{\begin{array}{l}
\dot{y} \\
\dot{x}
\end{array}\right\}+} \\
& {\left[\begin{array}{cc}
\frac{2 g}{L_{e}} & 0 \\
0 & \omega_{s}^{2}
\end{array}\right]\left\{\begin{array}{l}
y \\
x
\end{array}\right\}=-\left\{\begin{array}{c}
p \\
(1+\mu)
\end{array}\right\}}
\end{aligned}
$$

$$
c_{p}=\frac{\xi \sigma_{\dot{y}}}{\sqrt{2 \pi}}
$$

\section{Expresando (9) en forma matricial resulta:}

$$
\mathbf{M} \ddot{u}+\mathbf{C} \dot{u}+\mathbf{K} u=-\mathbf{M r} \ddot{u}_{g}
$$

La ecuación (11a) muestra el vector de grados de libertad $u$ y (11b) corresponde al vector de colocación del input.

$$
\ddot{y}+\frac{2 c_{p}}{L_{e}} \dot{y}+\frac{2 g}{L_{e}} y+p \ddot{x}=-p \ddot{u}_{g}
$$

donde $p=\frac{B_{h}}{L_{e}}$ se define como la razón de longitud (Chakraborty et al., 2012). Por otra parte, $\omega_{l}=\sqrt{\frac{2 g}{L_{e}}}$ es la frecuencia del líquido y $\gamma=\frac{\omega_{l}}{\omega_{s}}$ la razón de sintonía. Además, $\omega_{s}$ es la frecuencia desacoplada de la estructura principal.

\section{Ecuaciones de movimiento del sistema principal con ACLS}

La ecuación de movimiento de $M_{1}$ se muestra en la ecuación (5):

$$
\begin{aligned}
& \left(m_{s}+\rho A B_{h}+2 \rho h A\right) \ddot{x}+c_{s} \dot{x}+k_{s} x= \\
& -\left(m_{s}+\rho A B_{h}+2 \rho h A\right) \ddot{u}_{g}-\rho A B_{h} \ddot{y}
\end{aligned}
$$

donde $m_{1}=\rho A B_{h}+2 \rho h A$ y corresponde a la masa del líquido contenido en el ACLS. Remplazando en (5) se obtiene la ecuación (6):

$$
\left(m_{s}+m_{l}\right) \ddot{x}+c_{s} \dot{x}+k_{s} x=-\left(m_{s}+m_{l}\right) \ddot{u}_{g}-\rho A p L_{e} \ddot{y}
$$

Se define como la razón de masa del ACLS, el cuociente entre la masa del líquido y la masa del sistema principal:

$$
\mu=\frac{\rho A L_{e}}{m_{s}}
$$

Dividiendo (6) respecto a la masa del sistema principal $m_{\mathrm{s}}$ :

$$
(1+\mu) \ddot{x}+\frac{c_{s}}{m_{s}} \dot{x}+\frac{k_{s}}{m_{s}} x=-(1+\mu) \ddot{u}_{g}-\mu p \ddot{y}
$$

Las ecuaciones (4) y (8) pueden expresarse matricialmente de la siguiente forma:

$$
u=\left\{\begin{array}{ll}
x & y
\end{array}\right\}^{T} \quad \mathbf{r}=\left\{\begin{array}{ll}
0 & 1
\end{array}\right\}^{T}
$$

$\mathbf{M}$ es la matriz de masa, $\mathbf{C}$ es la matriz de amortiguamiento y K la matriz de rigidez del modelo $M_{1}$. Por otra parte, si planteamos el problema de segundo orden, por una formulación en espacio de estado de $M_{1}$. Entonces se define el vector de estado $\mathbf{X}_{1}$ como se muestra en la ecuación (12a):

$$
\mathbf{X}_{\mathbf{1}}=\left\{\begin{array}{llll}
x_{s} & y & \dot{x}_{s} & \dot{y}
\end{array}\right\}^{T}
$$

Con lo que se tiene la ecuación de estado (12b):

$$
\dot{\mathbf{X}}_{\mathbf{1}}=\mathbf{A} \mathbf{X}_{\mathbf{1}}+\mathbf{B}_{\mathbf{u}} \ddot{u}_{g}
$$

La matriz de estado del modelo $M_{1}$ se muestra en la ecuación (13a), el vector de colocación de excitación sísmica en la ecuación (13b) y la matriz de influencia del input en la ecuación (13c).

$$
\begin{array}{ll}
\mathbf{A}=\left[\begin{array}{cc}
0_{2 \times 2} & \mathrm{I}_{2 \times 2} \\
\mathbf{M}^{-1} \mathbf{K} & \mathbf{M}^{-1} \mathbf{C}
\end{array}\right] & \mathrm{B}_{\mathrm{u}}=\left[\begin{array}{c}
\mathbf{0}_{2 \times 1} \\
-\mathbf{M}^{-1} \mathbf{m}^{*}
\end{array}\right] \\
\mathrm{M}^{*}=\left[\begin{array}{cc}
1+\mu & 0 \\
0 & \mu
\end{array}\right] &
\end{array}
$$

\section{Optimización}

\section{Tipo de excitación sísmica}

Se realiza un análisis estocástico de sometido a una excitación sísmicas de alto contenido de frecuencias. El input sísmico se obtiene a través del ajuste de un filtro de Clough-Penzien (14), que se define como Densidad de 
Potencia Espectral $S_{g}(\omega)$, a un sismo derivado de la norma chilena NCh2745 (2013) para suelo tipo B.

$$
S_{g}(\omega)=S_{o} \frac{\omega_{g}^{4}+4 \xi_{g}^{2} \omega_{g}^{2} \omega^{2}}{\left(\omega_{g}^{2}-\omega^{2}\right)+4 \xi_{g}^{2} \omega_{g}^{2} \omega^{2}} \frac{\omega^{4}}{\left(\omega_{f}^{2}-\omega^{2}\right)+4 \xi_{f}^{2} \omega_{f}^{2} \omega^{2}}
$$

Por otra parte, $S_{o}$ es la intensidad del ruido blanco $\omega_{g}$, $\xi_{g}$, $\omega_{f}$ y $\xi_{f}$ son la frecuencia y amortiguamiento del estrato de suelo y del filtro, respectivamente. Estos parámetros se ajustan por mínimos cuadrados. Los valores de los parámetros del filtro se muestran en la Tabla 1.

\section{Tabla 1: Parámetros del filtro Clough-Penzien}

\begin{tabular}{|c|c|c|c|c|c|}
\hline \multirow{2}{*}{$\begin{array}{c}\text { Tipo de } \\
\text { entrada }\end{array}$} & \multicolumn{5}{|c|}{ Parámetros } \\
\cline { 2 - 6 } & $S_{o}$ & $\omega_{g}, \mathrm{rad} / \mathrm{s}$ & $\omega_{f}, \mathrm{rad} / \mathrm{s}$ & $\xi_{g}$ & $\xi_{f}$ \\
\hline BBP & 207.23 & 16.57 & 3.02 & 0.491 & 0.480 \\
\hline
\end{tabular}

BBP: Broad band process

El vector de estado del filtro, se define como (Saitua et al., 2018):

$$
\mathbf{X}_{\mathbf{f}}=\left\{x_{g} \dot{x}_{g} x_{f} \dot{x}_{f}\right\}^{T}
$$

Por otra parte, la ecuación de estado del filtro:

$$
\dot{\mathbf{X}}_{\mathbf{f}}=\mathbf{A}_{\mathbf{f}} \mathbf{X}_{\mathbf{f}}+\mathbf{B}_{\mathbf{f}} W(t) \quad \ddot{u}_{g}=\mathbf{C}_{\mathbf{f}} \mathbf{X}_{\mathbf{f}}
$$

donde $W(t)$ es la intensidad de ruido blanco que se calcula a partir de la relación (17):

$$
\sigma_{\ddot{u}_{g}}^{2}=\frac{P G A}{3}
$$

siendo $\sigma_{\ddot{u}_{g}}^{2}$ la varianza de la aceleración del suelo y $P G A$ (peak ground acceleration) la aceleración máxima del suelo, en esta investigación se utilizó $P G A=0.3 g$, donde $g$ es la aceleración de gravedad. Por otra parte, $\mathbf{A}_{\mathbf{f}}$ en la ecuación (18) es la matriz de estado del filtro, $\mathbf{C}_{\mathbf{f}}$ en (19a) es el vector de amortiguamiento del filtro, y $\mathbf{B}_{\mathbf{f}}$ en (19b) es el vector de colocación de excitación sísmica del filtro.

$$
\mathbf{A}_{\mathbf{f}}=\left[\begin{array}{cccc}
0 & 1 & 0 & 0 \\
-w_{g}^{2} & -2 \xi_{g} w_{g} 0 & 0 \\
0 & 0 & 0 & 1 \\
w_{g}^{2} & 2 \xi_{g} w_{g}-w_{f}^{2} & -2 \xi_{f} w_{f}
\end{array}\right]
$$

$$
\mathbf{C}_{\mathbf{f}}=\left[\begin{array}{ll}
w_{g}^{2} & 2 \xi_{g} w_{g}-w_{f}-2 \xi_{f} w_{f}
\end{array}\right] \quad \mathbf{B}_{\mathbf{f}}=\left[\begin{array}{c}
0 \\
-1 \\
0 \\
0
\end{array}\right]
$$

Entonces el vector y ecuación de estado del $\mathrm{M}_{1}$ se encuentran en las ecuaciones (20) y (21).

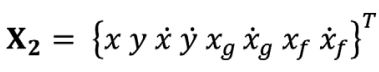

$$
\begin{aligned}
& \dot{\mathbf{X}}_{2}=\mathbf{A}_{2} \mathbf{X}_{2}+\mathbf{B}_{2} W(t)
\end{aligned}
$$

Y además, su matriz de estado y vector de colocación de excitación sísmica se muestran en las ecuaciones (22a) y (22b):

$$
A_{2}=\left[\begin{array}{cc}
A & B_{u} C_{f} \\
0 & A_{f}
\end{array}\right] \quad B_{2}=\left\{\begin{array}{c}
0 \\
B_{f}
\end{array}\right\}
$$

Ahora, expandiendo el estado, para incluir el filtro:

$$
\left\{\begin{array}{c}
\dot{\mathbf{X}}_{2} \\
\dot{\mathbf{X}}_{\mathbf{f}}
\end{array}\right\}=\left[\begin{array}{cc}
\mathbf{A} & \mathbf{B}_{\mathbf{u}} \mathbf{C}_{\mathbf{f}} \\
\mathbf{0} & \mathbf{A}_{\mathbf{f}}
\end{array}\right]\left\{\begin{array}{c}
\mathbf{X}_{2} \\
\mathbf{X}_{\mathbf{f}}
\end{array}\right\}+\left\{\begin{array}{c}
\mathbf{0} \\
\mathbf{B}_{\mathbf{f}}
\end{array}\right\} W(t)
$$

\section{Incertidumbre en los parámetros}

Los parámetros considerados para incluir una incertidumbre positiva y negativa de 5 y $10 \%$ fueron la razón de longitud $p$, razón de masa $\mu$ y el periodo $T_{s}$. Se consideraron 3 tipos diferentes de $1.5,2.0$ y $2.5 \mathrm{~s}$, correspondientes a estructuras rígidas, semi-flexibles y flexibles, respectivamente, con el objetivo de analizar las estructuras más comunes. Para introducir incertidumbre en los parámetros, se obtiene la matriz de covarianza a través de la resolución de la ecuación de Lyapunov, expandida a través de una serie de Taylor, hasta primer orden (ecuación (25)), donde $R$ corresponde a la matriz de covarianza del sistema completo, sin incertidumbre. Como resultado, se tiene la ecuación de Lyapunov sin considerar incertidumbre:

$$
\overline{\mathbf{A}_{\mathbf{1}} \mathbf{R}}+{\overline{\mathbf{R} \mathbf{A}_{1}}}^{\mathbf{T}}+\overline{\mathbf{B}_{1}} W=0
$$

Para agregarle la incertidumbre, se deriva la variación con respecto al parámetro $x_{i}$ :

$$
\overline{\mathbf{A}_{\mathbf{1}}} \frac{\partial R}{\partial x_{i}}+\overline{\mathbf{R}} \frac{\partial A_{1}}{\partial x_{i}}+\overline{\mathbf{R}} \frac{\partial A_{1}^{T}}{\partial x_{i}}+\mathbf{A}_{\mathbf{1}}^{\mathrm{T}} \frac{\partial R}{\partial x_{i}}+\overline{\mathbf{B}_{\mathbf{1}}} W=0
$$

donde $x_{i}=\left\{p, \mu, T_{s}\right\} \quad$ son los parámetros a los que se le agrega incertidumbre. Para agregar la incertidumbre 
positiva y negativa, se calcula la desviación estándar de la media cuadrática, donde la media cuadrática en nuestra investigación es la desviación estándar de desplazamiento lateral de la estructura $\sigma_{x}$ quedando nuestro sistema de incertidumbre de parámetros como sigue (Debbarma et al., 2010):

$$
\sigma_{x}=\bar{\sigma}_{x} \pm \delta x_{i} \pm \ldots \sum_{i=1}^{m} \frac{\partial \sigma_{x}}{\partial x_{i}}\left\{\begin{array}{l}
\sigma_{x u p}=\bar{\sigma}_{x}+\sum_{i=1}^{m} \frac{\partial \sigma_{x}}{\partial x_{i}} \pm \ldots \\
\sigma_{x l o w}=\bar{\sigma}_{x}-\sum_{i=1}^{m} \frac{\partial \sigma_{x}}{\partial x_{i}} \pm \ldots
\end{array}\right.
$$

\section{Función objetivo}

El problema de optimización a resolver es un problema de MIN-MAX, en el cual se requiere determinar la razón de sintonía $\gamma_{o p t}$ y el coeficiente de pérdida de carga $\xi_{\text {opt }}$ del sistema con ACLS, considerando incertidumbre en los parámetros del sistema. De esta manera, la optimización se puede representar del siguiente modo:

$$
\begin{aligned}
\text { Minimizar: } & J\left(\gamma_{o p t \_u p}, \xi_{o p t \_u p}\right)=\operatorname{Min}\left(\sigma_{x_{s} u p}\right) \\
& J\left(\gamma_{o p t}, \xi_{\text {opt }}\right)=\operatorname{Min}\left(\sigma_{x_{s}}\right) \\
& J\left(\gamma_{\text {opt_low }}, \xi_{\text {opt_low }}\right)=\operatorname{Min}\left(\sigma_{x_{s} l o w}\right)
\end{aligned}
$$

Con los siguientes límites de optimización:

$$
0.5<\gamma_{l}<1.5 \quad 0<\xi_{l}<30
$$

donde $\sigma_{x u p}$ y $\sigma_{x l o w}$ son las desviaciones estándar del desplazamiento en $x$ considerando incertidumbre positiva y negativa respectivamente. Por otra parte, $\sigma_{x s}$ es la desviación estándar del desplazamiento en $x$ sin incertidumbre.

El movimiento del líquido de columna del amortiguador no debe sobrepasar el nivel de altura de la columna del ACLS, evitando el derrame. Para lo cual se debe cumplir la ecuación (29). Por otra parte, $c$ es el peak factor que maximiza la desviación estándar de desplazamiento vertical de la superficie libre del líquido, hasta una desviación estándar máxima.

$$
h-c \sigma_{y} \geq 0
$$

Donde $c$ se consideró como 2, de acuerdo a estudios realizados anteriormente (Espinoza et al., 2018).

\section{Eficiencia del ACLS}

La eficiencia del ACLS, cuando se ha introducido incertidumbre, se calculó de acuerdo a la ecuación (30), donde $R D$ es una eficiencia porcentual, es decir, $R D$ es nulo cuando la eficiencia es nula, y es 100 , cuando la eficiencia es máxima.

$R D=\left(1-\frac{\sigma_{x c / A C L S}}{\sigma_{x s / A C L S}}\right) \cdot 100 \%$

Donde $\sigma_{x c / A C L S}$ y $\sigma_{x s / A C L S}$ corresponden a la desviación estándar del desplazamiento del sistema principal controlado por un ACLS óptimo y sin ACLS, respectivamente.

\section{Resultados}

A continuación se entrega la sensibilidad en los parámetros óptimos señalados anteriormente. Las incertidumbres en los parámetros considerados son de $\pm 5 \mathrm{y} \pm 10 \%$.

\section{Sensibilidad de los parámetros óptimos del TLCD \\ Influencia de la incertidumbre de la razón de masa}

La Figura 2 muestra 6 gráficos, en donde la fila inferior corresponde al coeficiente de pérdida de carga óptimo $\xi_{\text {opt }}$, y la superior corresponde a la razón de sintonía $\gamma_{o p t}$. Estos gráficos se encuentran en función de la razón de masa $\mu_{l}$ y para un amortiguamiento del sistema $\xi_{s}=0.05$. Cada columna corresponde a un periodo de la estructura, desde estructuras más rígidas (izquierda) a más flexibles hacia la derecha. Se observa, que la razón de frecuencias posee un decrecimiento lineal con respecto a la razón de masa para los tres periodos. En tanto, el coeficiente de pérdida de carga tiende a aumentar en forma lineal a medida que aumenta la razón de masa, además este incremento es mayor a medida que la estructura es más flexible.

Por otra parte, se aprecia que la incertidumbre aumenta a medida que incrementa $\mu_{l}$ para ambos parámetros óptimos. Se observa un comportamiento distinto para estructuras rígidas respecto a las flexibles. Con respecto a las rígidas, la razón de frecuencias aumenta a medida que el porcentaje de incertidumbre disminuye, y para las flexibles disminuye, a medida que el porcentaje de incertidumbre aumenta. Por otra parte, el comportamiento del coeficiente de pérdida 

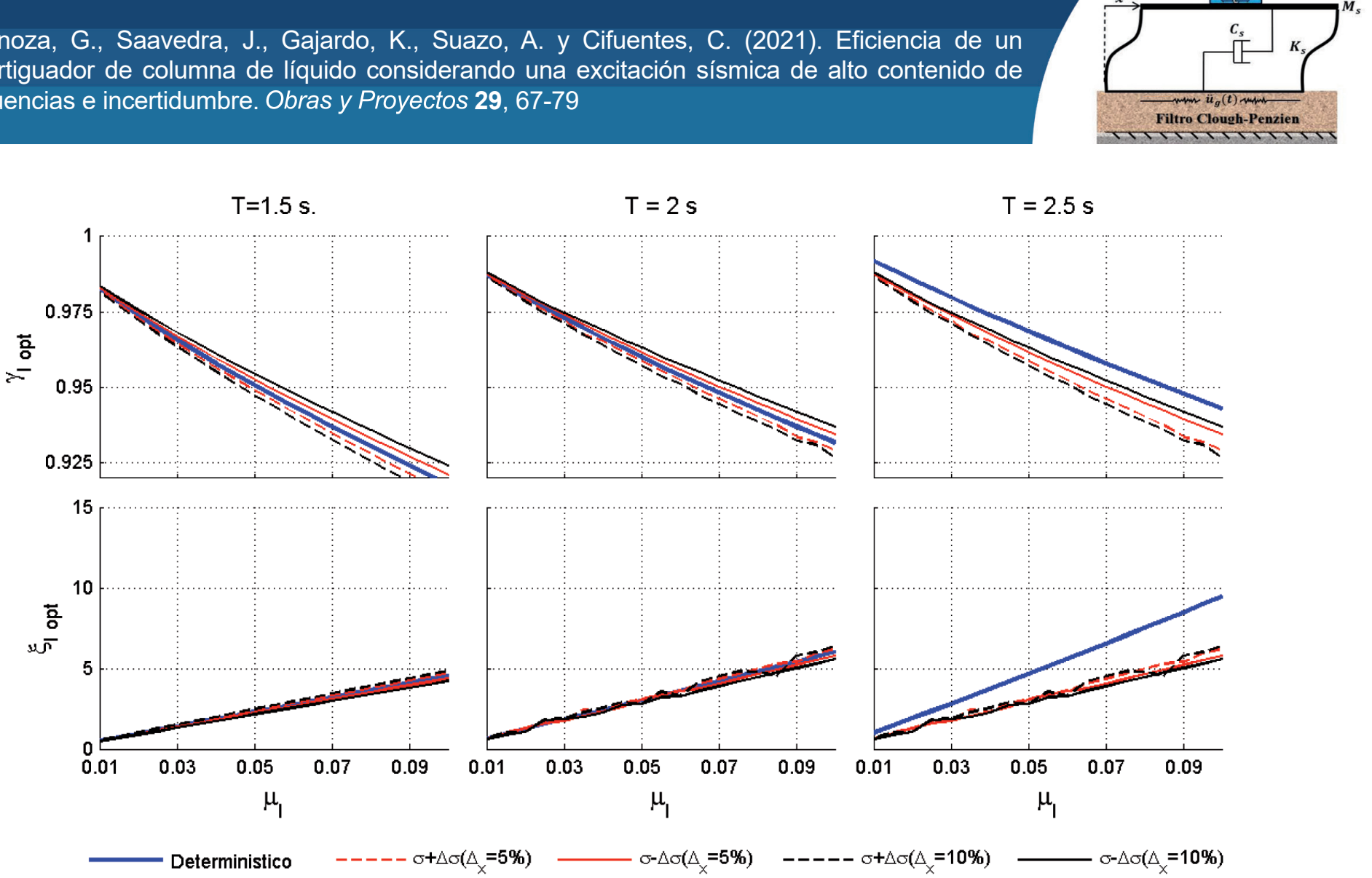

Figura 2: Parámetros óptimos del ACLS con incertidumbre en la razón de masa de $\pm 5 \mathrm{y} \pm 10 \%$; $\xi_{\mathrm{s}}=0.05$
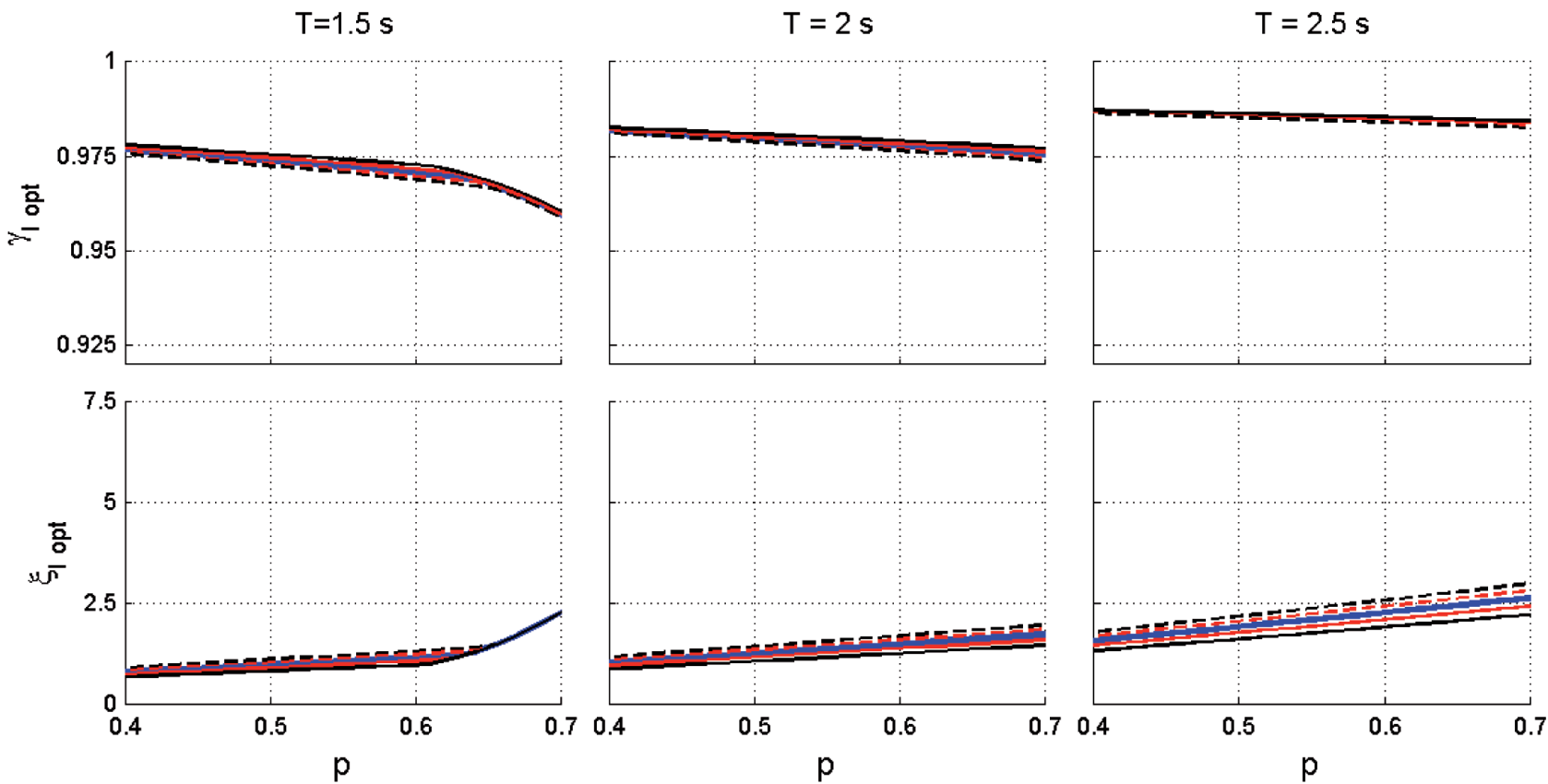

Deterministico

$-----\sigma+\Delta \sigma\left(\Delta_{x}=5 \%\right)$

$\sigma-\Delta \sigma\left(\Delta_{x}=5 \%\right)$

$-----\sigma+\Delta \sigma\left(\Delta_{x}=10 \%\right)$ $\sigma-\Delta \sigma\left(\Delta_{x}=10 \%\right)$

Figura 3: Parámetros óptimos del ACLS con incertidumbre en la razón de longitud de $\pm 5 \mathrm{y} \pm 10 \% ; \xi_{\mathrm{s}}=0.05$ 
de carga es opuesto al de la razón de frecuencias. En el caso de estructuras flexibles, se observa que para todas las incertidumbres, tanto positivas como negativas, el valor de los parámetros óptimos disminuye.

\section{Influencia de la incertidumbre en la razón de longitud}

La Figura 3 muestras los parámetros óptimos del ACLS, en función de la razón de longitud, con porcentajes de incertidumbre de $\pm 5 \% \mathrm{y} \pm 10 \%$. Los gráficos se distribuyen en forma análoga a la Figura 2. Al igual que en la Figura 2, el comportamiento de los parámetros óptimos del ACLS en estructuras más rígidas, es muy distinto al de las estructuras más flexibles. Se observa que la razón de sintonía óptima, tiende a ser insensible en estructuras flexibles. En estructuras con $T_{s}=2 \mathrm{~s}$ disminuye levemente. En estructuras rígidas, se produce un decrecimiento en forma cuadrática. Por otra parte, en estructuras flexibles e intermedias, el coeficiente de pérdida de carga se incrementa en forma lineal. En el caso de estructuras más rígidas, el coeficiente de pérdida de carga crece en forma cuadrática.

Con respecto a la incertidumbre, el valor óptimo del coeficiente de pérdida de carga en estructuras flexibles y semiflexibles, es más sensible respecto a una incertidumbre en la razón de longitud. Además, una incertidumbre positiva en la razón de longitud, produce un aumento del coeficiente de pérdida de carga, y una negativa, lo opuesto.

\section{Influencia de la incertidumbre en el periodo de la estructura principal}

La Figura 4 muestra la razón de sintonía $\gamma_{\text {opt }}$ y el coeficiente de pérdida de carga $\xi_{\text {opt }}$ óptimos en función del período de la estructura principal $T_{s}$, para un amortiguamiento del sistema $\xi_{s}=0.05$. Cada columna considera una razón de masa del líquido, columna izquierda $2 \%$, intermedia $3 \%$ y derecha $5 \%$. Se observa, que tanto la razón de frecuencias como el coeficiente de pérdida de carga aumentan a medida que la estructura es más flexible, siendo mayor a medida que aumenta la razón de masa. Existe una gran sensibilidad en la razón de frecuencia óptima del ACLS a la incertidumbre en el periodo de la estructura, la que disminuye a medida que la estructura es más flexible. Se observan variaciones en la razón de frecuencia óptima de un 5 a 7\%. Con respecto a la sensibilidad del coeficiente de pérdida de carga óptimo a la inclusión de incertidumbre en el periodo, se observa que es muy pequeña y aumenta con la razón de masa.

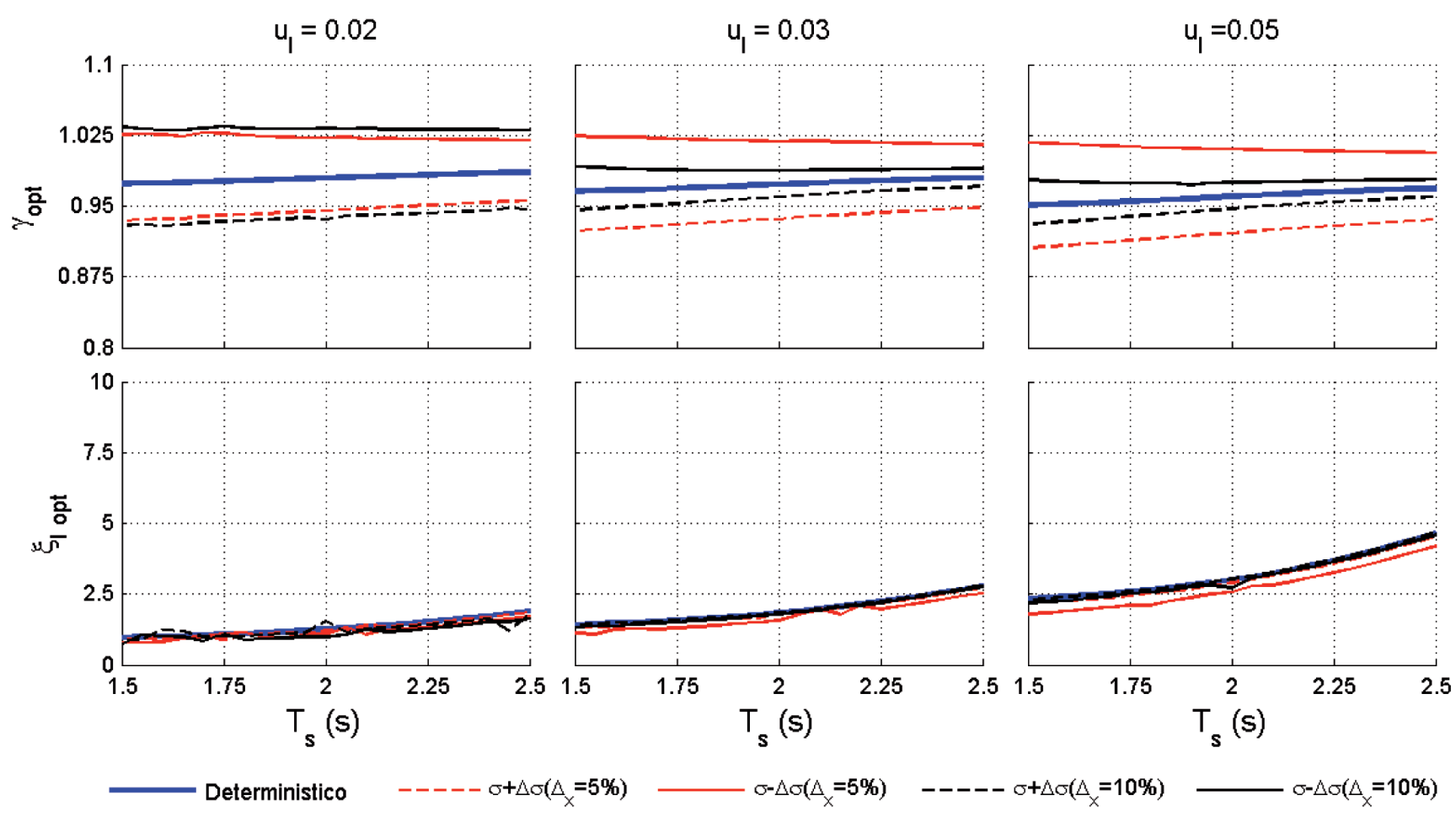

Figura 4: Parámetros óptimos del ACLS con incertidumbre en el periodo de la estructura principal de $\pm 5 \mathrm{y} \pm 10 \%$; $\xi_{\mathrm{s}}=0.05$ 


\section{Ẹiciencia respecto a parámetros sistema}

A continuación se estudia la sensibilidad en la eficiencia del ACLS, cuando se introduce incertidumbre en los parámetros. Esta se calcula como un porcentaje de reducción de la desviación estándar de desplazamiento de la estructura con un ACLS, con respecto a uno sin ACLS (ecuación (30)).

\section{Sensibilidad de la eficiencia respecto a la razón de masa}

En la Figura 5 se entrega la eficiencia del ACLS, cuando varía la razón de masa desde un 1 a $10 \%$. Cada columna corresponde a tres periodos de la estructura $T_{s}=1.5,2.0$ y $2.5 \mathrm{~s}$. La Figura 5 muestra a medida que la razón de masa es mayor, la eficiencia es mayor. Por otra parte, se observa prácticamente una insensibilidad a un ingreso de incertidumbre en la razón de masa.
Sensibilidad de la eficiencia respecto a la razón de longitud

En la Figura 6 se entrega la eficiencia del ACLS en función de la razón de longitud. Para estructuras rígidas, intermedias y flexibles. Cuando existe una incertidumbre de $\pm 5 \mathrm{y} \pm 10 \%$. Se observa para todos los periodos, que la eficiencia aumenta en forma lineal, a medida que aumenta la razón de longitud. También se observa que la eficiencia es independiente de la incertidumbre en la razón de longitud.

\section{Sensibilidad de la eficiencia respecto del periodo}

La Figura 7 muestra la eficiencia del ACLS en función de la flexibilidad de estructura principal. Esta también se entrega para tres razones de masa, una para cada columna. Las razones de masa aumentan de izquierda a derecha. Se observa una mayor sensibilidad de la eficiencia, para razones de masa menores. Por otra parte, la sensibilidad de la eficiencia con respecto a una incertidumbre en el periodo
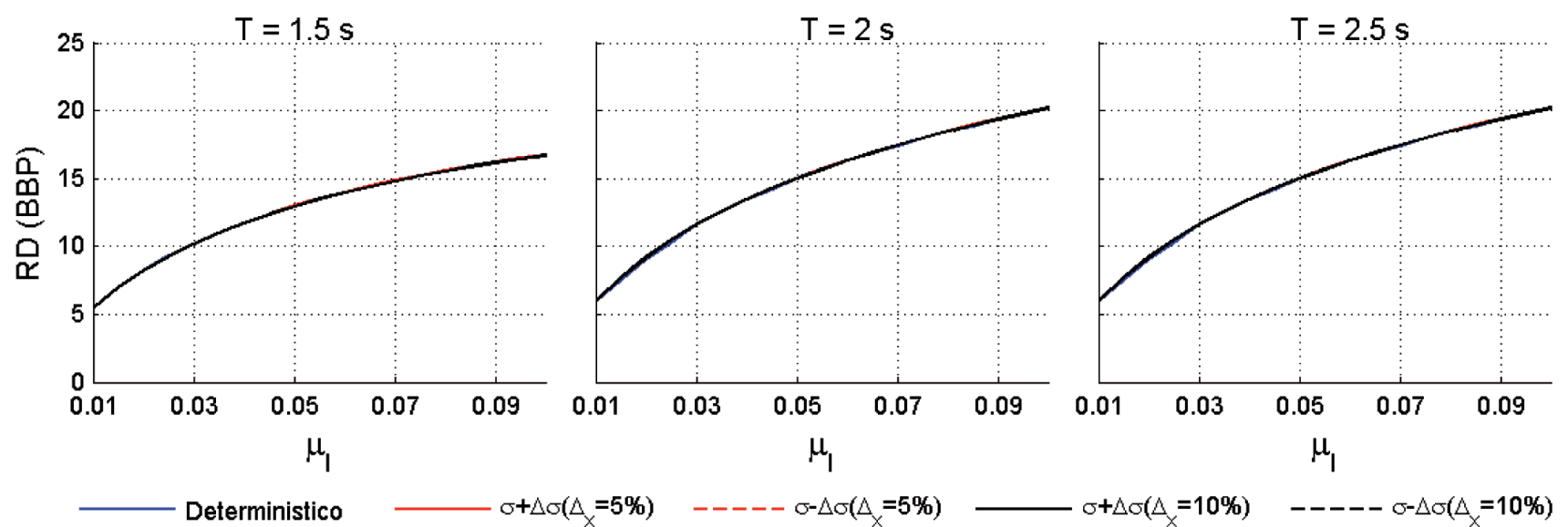

Figura 5: Porcentaje de reducción del desplazamiento de la estructura con un ACLS óptimo con incertidumbre en la razón de masa de $\pm 5 \mathrm{y} \pm 10 \% ; \xi_{\mathrm{s}}=0.05$
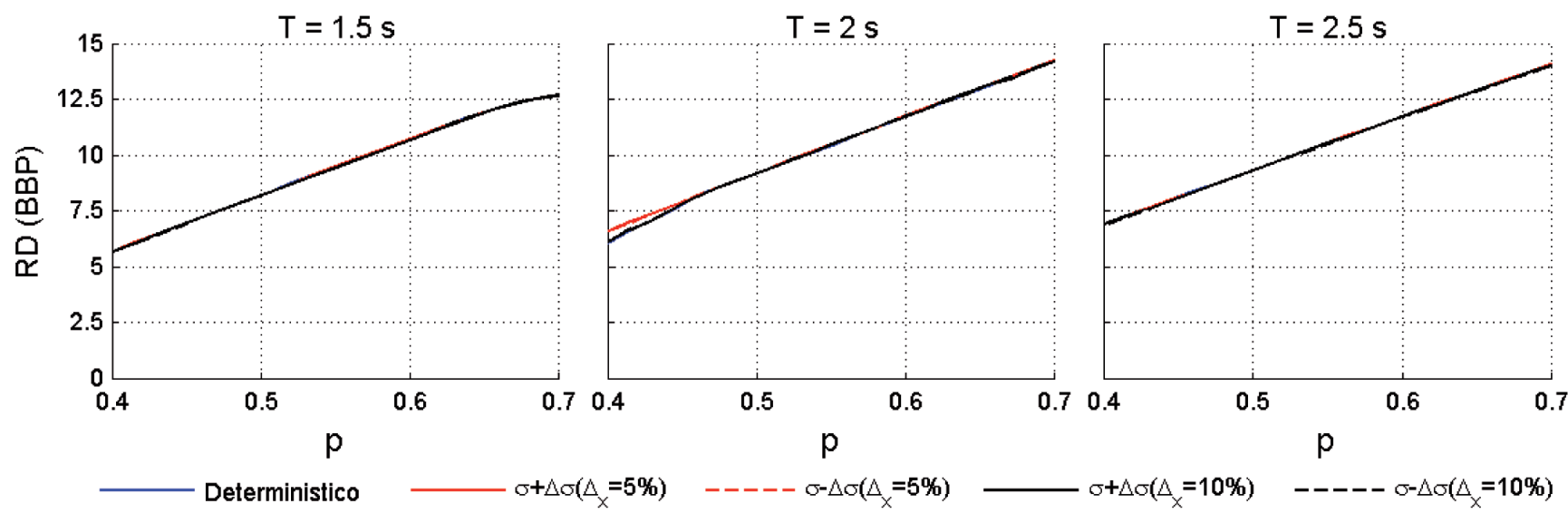

Figura 6: Porcentaje de reducción del desplazamiento de la estructura con un ACLS óptimo, con incertidumbre en la razón de longitud de $\pm 5 \mathrm{y} \pm 10 \% ; \xi_{\mathrm{s}}=0.05$ 
disminuye con respecto a la flexibilidad de la estructura. Además, la Figura 7 muestra que para cualquier porcentaje de incertidumbre en el periodo, la eficiencia es mayor.

\section{Raíz cuadrática media RMS del despla- zamiento de la estructura}

A continuación se muestra la raíz cuadrática media RMS del desplazamiento del sistema principal, controlado por un ACLS óptimo, con respecto a parámetros del sistema, cuando se ha incluido una incertidumbre en ellos.

Influencia en la RMS de una incertidumbre en la razón de masa

En la Figura 8 se observa la RMS en función de la razón de masa, cada columna corresponde a una flexibilidad distinta, desde izquierda a derecha $T_{s}=1.5,2.0$ y $2.5 \mathrm{~s}$. La Figura 8 muestra una leve disminución del RMS respecto a la razón de masa. Por otro lado, también se observa un aumento del desplazamiento con respecto a la flexibilidad de la estructura. En cuanto a la inclusión de incertidumbre en la razón de masa, no se observa ningún cambio en el RMS, con respecto al porcentaje de incertidumbre.

\section{Influencia en la RMS de la razón de longitud}

La Figura 9 muestra la influencia de la incertidumbre en la razón de longitud en la RMS del desplazamiento de la estructura. Este resultado se entrega en función en función de la razón de longitud. Se analizan tres periodos de la estructura principal de $1.5,2.0$ y $2.5 \mathrm{~s}$, es decir, estructuras más rígidas a más flexibles. Se observa que la RMS del
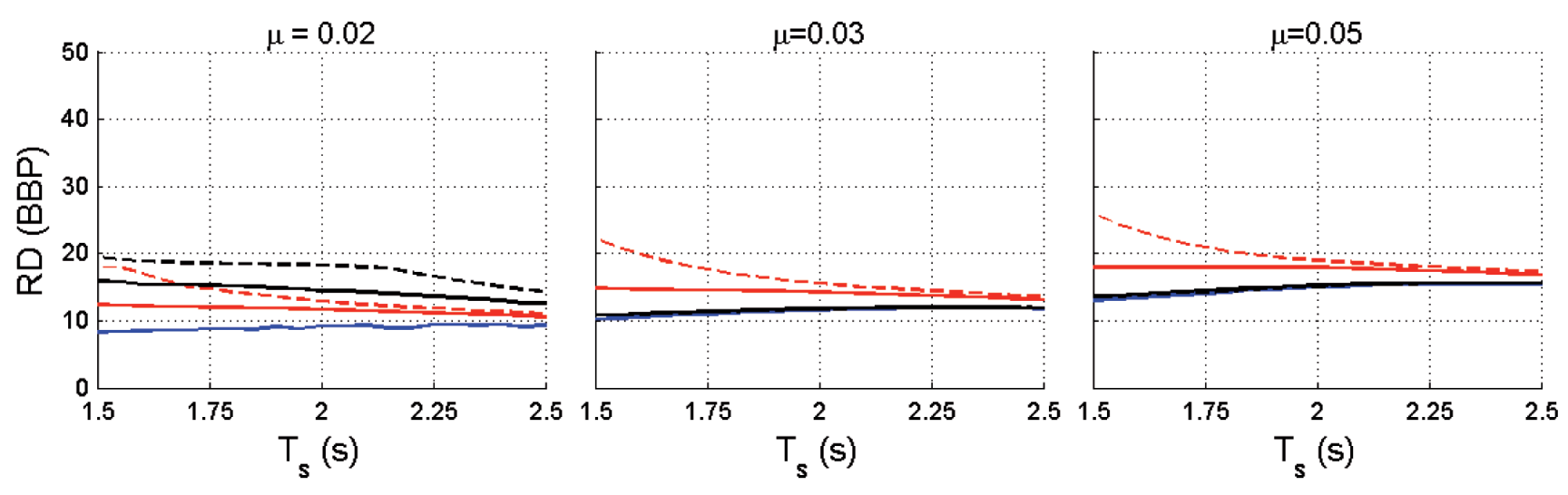

Deterministico

$\sigma+\Delta \sigma\left(\Delta_{x}=5 \%\right)$

$-----\sigma-\Delta \sigma\left(\Delta_{\times}=5 \%\right)$

$\sigma+\Delta \sigma\left(\Delta_{X}=10 \%\right)$

$-----\sigma-\Delta \sigma\left(\Delta_{x}=10 \%\right)$

Figura 7: Porcentaje de reducción del desplazamiento de la estructura con un ACLS óptimo con incertidumbre en el periodo de la estructura de $\pm 5 \mathrm{y} \pm 10 \% ; \xi_{\mathrm{s}}=0.05$

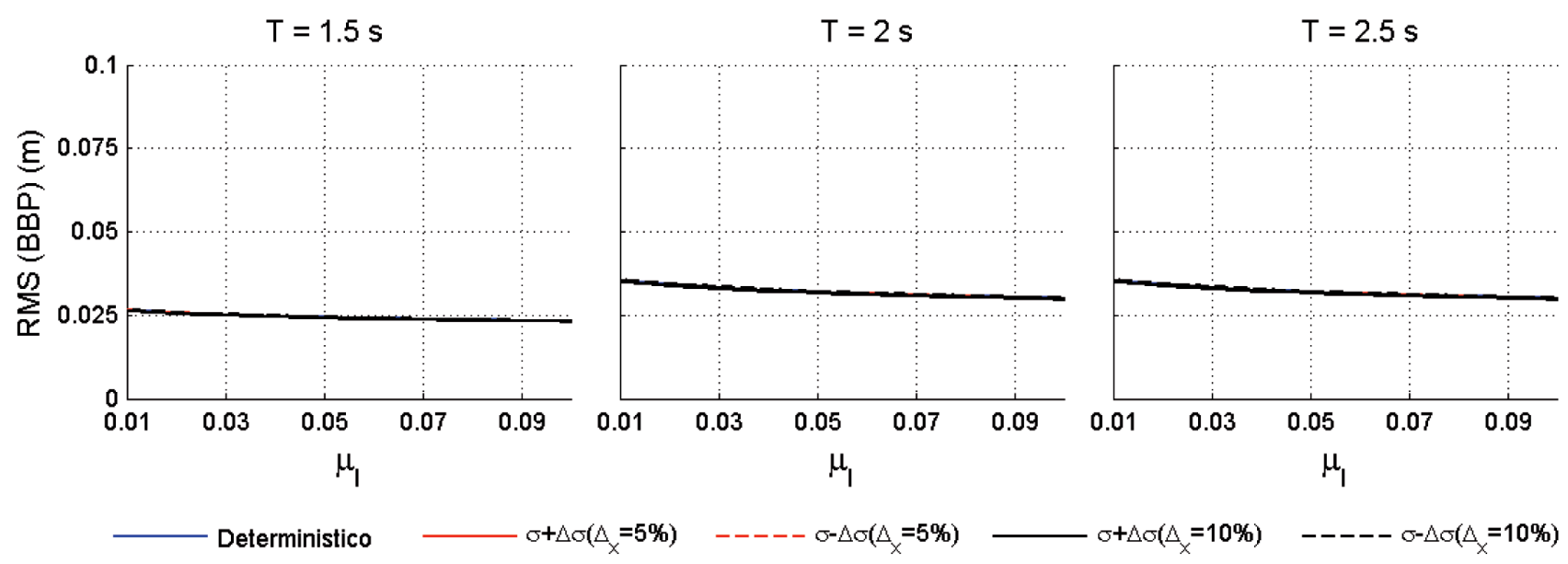

Figura 8: RMS del desplazamiento de la estructura con un ACLS óptimo con incertidumbre en la razón de masa de $\pm 5 \mathrm{y} \pm 10 \%$; $\xi_{\mathrm{s}}$ $=0.05$ 
desplazamiento de la estructura, es insensible a la razón de longitud y aumenta con la flexibilidad de la estructura.

\section{Influencia en la RMS del periodo de la estructura principal}

A continuación se encuentra la Figura 10 que entrega la RMS de desplazamiento de la estructura principal, en función del periodo de la estructura, para tres razones de masa.

Se observa un aumento asintótico del RMS a medida que aumenta el periodo de la estructura. Por otra parte, la Figura 10 muestra que existe una mayor sensibilidad a la introducción de incertidumbre, para razones de masa menores. Y además hay una mayor sensibilidad en estructuras más rígidas. Además, se observa que, para razones de masa pequeñas, la RMS del desplazamiento aumenta para incertidumbres positivas y disminuye para incertidumbres negativas. En el caso de razones de masa mayores, se produce el mismo efecto que en el caso anterior, pero para mayores incertidumbres, la variación del RMS es menor.

\section{Conclusiones}

De los resultados de la investigación, se concluye:

1. Existe una gran sensibilidad de los parámetros de diseño del ACLS cuando se introduce incertidumbre en la razón masa, la que aumenta a medida que aumenta ésta.

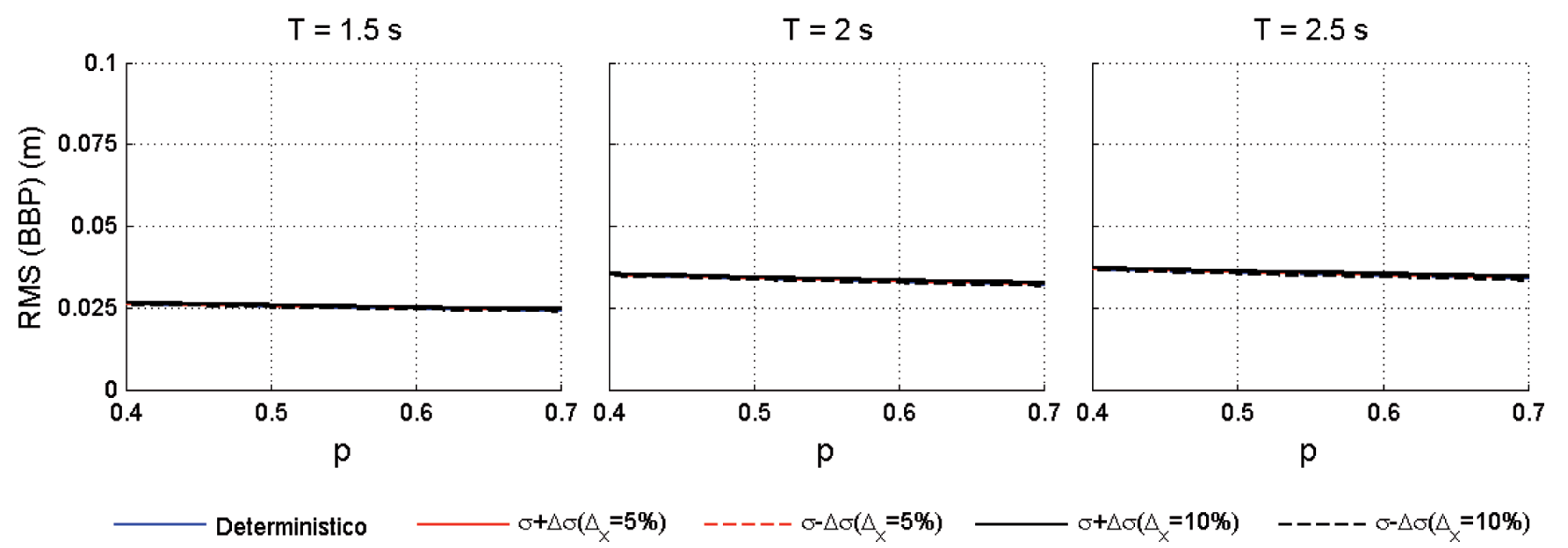

Figura 9: Porcentaje de reducción del desplazamiento de la estructura con un ACLS óptimo, con incertidumbre en la razón de longitud de un $\pm 5 \mathrm{y} \pm 10 \% ; \xi_{\mathrm{s}}=0.05$
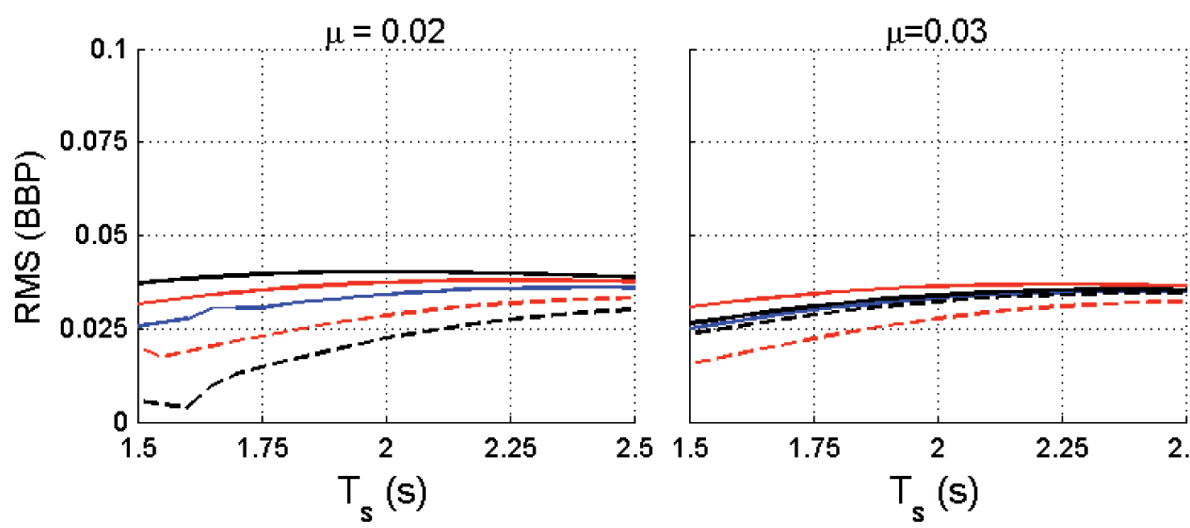

Deterministico

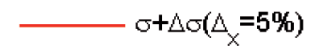

$-----\sigma-\Delta \sigma\left(\Delta_{X}=5 \%\right)$

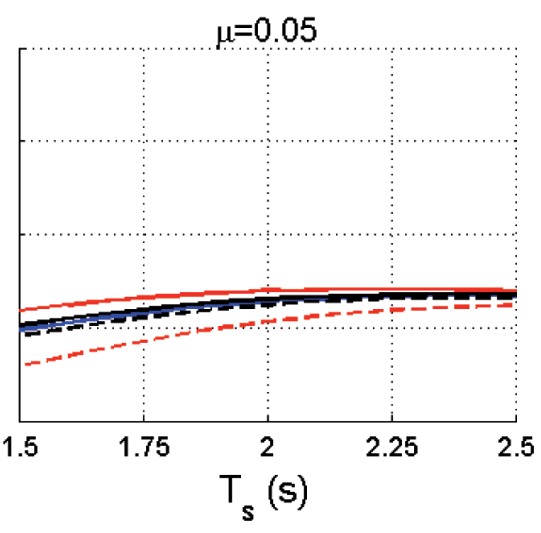

$\sigma+\Delta \sigma\left(\Delta_{x}=10 \%\right) \quad-----\sigma-\Delta \sigma\left(\Delta_{x}=10 \%\right)$

Figura 10: RMS del desplazamiento de la estructura con un ACLS óptimo con incertidumbre en el periodo de la estructura de \pm 5 y $\pm 10 \% ; \xi_{\mathrm{s}}=0.05$ 
2. Existe una gran sensibilidad del coeficiente de pérdida de carga óptimo, al introducir incertidumbre en la razón de longitud del ACLS, la que es mayor para razones de longitud mayores, y estructuras flexibles.

3. La introducción de incertidumbre en el periodo de la estructura produce una gran variación en el valor de la razón de frecuencias óptima del ACLS.

4. Una incertidumbre en el periodo de la estructura produce una variación importante en la reducción de desplazamiento, en estructuras con razones de masa menores y periodos mayores.

5. Existe una gran sensibilidad de la RMS de desplazamiento ante una incertidumbre en el periodo de la estructura principal, sobretodo en estructuras rígidas y con razones de masa menores del ACLS.

\section{Agradecimientos}

Los autores agradecen a la Universidad del Bío-Bío, por el financiamiento otorgado para el desarrollo de esta investigación, a través de Proyecto Regular de Investigación DIUBB 2060542 IF/R.

\section{Referencias}

Balendra, T., Wang C.M. and Cheong, H.F. (1995). Effectiveness of tuned liquid column dampers for vibration control of towers. Engineering Structures 17(9), 668-675

Chakraborty, S., Debbarma, R. and Marano, G.C. (2012). Performance of tuned liquid column dampers considering maximum liquid motion in seismic vibration control of structures. Journal of Sound and Vibration 331(7), 1519-1531

Debbarma, R., Chakraborty, S., and Ghosh, S.K. (2010) Optimum design of tuned liquid column dampers under stochastic earthquake load considering uncertain bounded system parameters. International Journal of Mechanical Sciences 52(10), 1385-1393

Di Matteo, A., Lo Iacono, F., Navarra, G. and Pirrotta, A. (2015). Optimal tuning of tuned liquid column damper systems in random vibration by means of an approximate formulation. Meccanica 50(3), 795-808
Espinoza, G., Quinteros, C., Gajardo, K., Suazo, A. y Quijada, S. (2021). Eficiencia de un amortiguador de columna de líquido sintonizado considerando una excitación sísmica de bajo contenido de frecuencias e incertidumbre. Obras y Proyectos 29, $54-66$

Espinoza, G., Sagredo, G. y Suazo, A. (2020a). Análisis de la eficiencia de un amortiguador combinado sintonizado con incertidumbre en los parámetros sometido a una excitación sísmica de alto contenido de frecuencias. Obras y Proyectos 28, 58-67

Espinoza, G., Rivas, S. y Suazo, A. (2020b). Análisis de la eficiencia de un amortiguador combinado sintonizado con incertidumbre en los parámetros sometido a excitaciones sísmicas de bajo contenido de frecuencias. Obras y Proyectos 28, 68-77

Espinoza, G., Carrillo, C. and Suazo, A. (2018). Analysis of a tuned liquid column damper in non-linear structures subjected to seismic excitations. Latin American Journal of Solids and Structures 15(7), e91

Lee, S.K., Lee, H.R. and Min, K.W. (2010). Experimental verification on nonlinear dynamic characteristic of a tuned liquid column damper subjected to various excitation amplitudes. The Structural Design of Tall and Special Buildings 21(5), 374-388

NCh2745 (2013). Análisis y diseño de edificios con aislación sísmica. Instituto Nacional de Normalización INN, Santiago, Chile

NCh433 (2009). Diseño sísmico de edificios. NCh433 Of.1996 mod.2009. Instituto Nacional de Normalización INN, Santiago, Chile

Pabarja, A., Vafaei, M., Alih, S.C., Md Yatim, M.Y. and Osman, S.A. (2019). Experimental study on the efficiency of tuned liquid dampers for vibration mitigation of a vertically irregular structure. Mechanical Systems and Signal Processing 114, 84-105 Rozas, L., Boroschek, R.L., Tamburrino, A. and Rojas, M. (2016). A bidirectional tuned liquid column damper for reducing the seismic response of buildings. Structural Control and Health Monitoring 23(4), 621-640

Saitua, F., Lopez-Garcia, D. and Taflanidis, A.A. (2018). Optimization of height-wise damper distributions considering practical design issues. Engineering Structures 173,768-786 
Sakai, F., Takaeda, S. and Tamaki, T. (1989). Tuned liquid column damper - New type device for suppression of building vibrations. First International Conference on High-Rise Buildings, Nanjing, China, 926-931

Shum, K.M. and Xu, Y.L. (2002). Multiple-tuned liquid column dampers for torsional vibration control of structures: experimental investigation. Earthquake Engineering and Structural Dynamics 31(4), 977-991

Shum, K.M. (2009). Closed form optimal solution of a tuned liquid column damper for suppressing harmonic vibration of structures. Engineering Structures 31(1), 84-92
Zhu, F., Wang, J.T., Jin, F. and Altay, O. (2015). Real-time hybrid simulation of single and multiple tuned liquid column dampers for controlling seismic-induced response. $6^{\text {th }}$ International Conference on Advances in Experimental Structural Engineering, University of Illinois, Urbana-Champaign, USA 